\title{
The Effect of Orifice Diameter to the Acoustic Signals Captured at the Cold Part of a Ranque-Hilsch Vortex Tube
}

\author{
Wirachman Wisnoe ${ }^{1}$, Khairil Muhaimin Abd Rahman ${ }^{1}$, Yusman Istihat ${ }^{2}$ and Valliyappan David Natarajan ${ }^{1}$ \\ ${ }^{1}$ Faculty of Mechanical Engineering, Universiti Teknologi MARA (UiTM), 40450 Shah Alam, Malaysia \\ ${ }^{2}$ Faculty of Engineering, Universiti Selangor (UNISEL), 45600 Bestari Jaya, Malaysia
}

\begin{abstract}
In this study, acoustic analysis and thermofluid performance of a Ranque-Hilsch Vortex Tube (RHVT) is experimentally investigated under different orifice diameters at its cold tube. The orifice diameters used are $2 \mathrm{~mm}$, $3 \mathrm{~mm}, 4 \mathrm{~mm}, 5 \mathrm{~mm}$ and $6 \mathrm{~mm}$. The inlet pressure (gage) is set at $10 \mathrm{psi}, 15 \mathrm{psi}, 20 \mathrm{psi}$ and $25 \mathrm{psi}$ for each orifice diameter. The sound produced by the tube is recorded using a microphone located outside the cold tube. The acoustic signal is processed using Fast-Fourier Transform (FFT) to obtain the frequency representation. Main frequencies are then extracted to constitute the signature of the signal for that specific configuration. It is observed that different orifice diameters give different signatures. These signatures are then associated with the thermofluid performance of the device to obtain the relation among the parameters.
\end{abstract}

\section{Introduction}

Ranque-Hilsch Vortex Tube (RHVT) (see figure 1) is an industrial device that is able to produce hot and cold temperatures of air flow from compressed air. Compressed air supplied at the inlet of the device enters several nozzles at a swirl generator to create swirl motion. This swirl flow moves close to the tube wall towards the hot outlet and exits through the conical valve. The centre part of the flow in the tube is bounced back creating a secondary flow towards the orifice and exits through the cold outlet of the device. The flow of air leaving the conical valve has a higher temperature relative to the inlet temperature, while the flow leaving the orifice has a lower temperature relative to the inlet temperature.

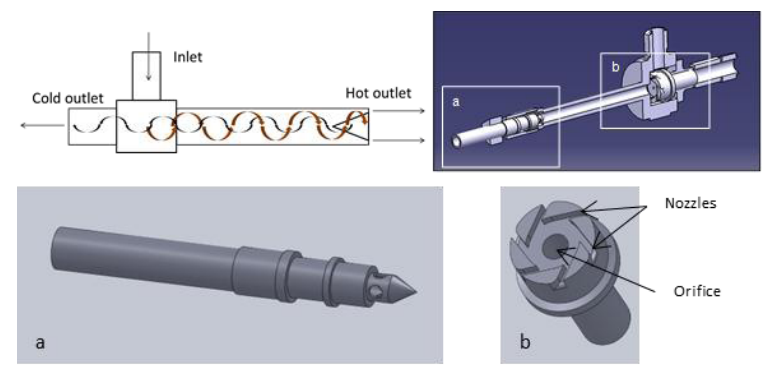

Figure 1. RHVT and its components. (a) Conical valve and (b) Swirl generator with nozzles and orifice.

Despite its simplicity of mechanism, the phenomenon is not well understood. Throughout the years, many researches have been done in order to explain the phenomena. Universiti Teknologi MARA (UiTM) Malaysia has started the research on RHVT since 2011.
The study covered experimental, CFD simulation, visualization and acoustic analysis. The study conducted by Ismail et al. [1] revealed the influence of parameters to the overall performance of an RHVT. They conducted experiments by varying the inlet pressure, nozzle depth and orifice diameter. They found that the increase of inlet pressure and nozzle depth would improve the performance of the device. However, the performance of the device was not in linear relation with the orifice diameter. This experimental study is also done by other researchers such as Thakera [2] and Nimbalkar [3]. The visualisation of the device was done by Istihat et al. [4] using a transparent tube with bigger scale from the actual RHVT. Through this visualisation they could observe the swirl motion inside the tube. Wisnoe et al. [5] conducted experiments to associate the effect of different nozzles depth to the acoustic signals produced by the RHVT, as also suggested by Kurosaka [6] about the acoustic streaming of the device. They observed that increasing the inlet pressure did not displace the signal frequencies significantly when the same swirl generator was used within the range of inlet pressures studied. The magnitude of the frequencies, however, changed significantly when different swirl generator was used. Different swirl generator produced different set of frequencies. The set of frequencies-magnitudes represent unique signature of the RHVT for that specific configuration and performance of the RHVT. Abd Rahman et al. [7] further investigated the acoustic signatures produced by the RHVT by studying the effect of orifice diameter to the acoustic signature at the hot tube. It was observed that the acoustic signature remained 
unchanged when different orifice diameters were used. In other study, Istihat et al. [8] presented the wavelet transform of the acoustic signals recorded at the outlet of cold and hot tubes. The wavelet analysis allowed obtaining the time-frequency representation of the signals.

This paper is focusing on the effect of the orifice diameter to the acoustic signal produced at the cold side of the tube and to its thermofluid performances.

\section{Methodology}

Fig. 2 shows the schematic diagram of the experiment. This experiment setting is to ensure the thermofluid data (mass flow rate, pressure and temperature) at the inlet and both outlets are measured properly and the sound produced at the cold tube of the RHVT is captured. An "anechoic" box (acoustic box) was used to isolate the sound produced by the tube from the surrounding noise. The sound from tube was captured by a B\&K microphone connected to a multichannel spectrum analyzer.

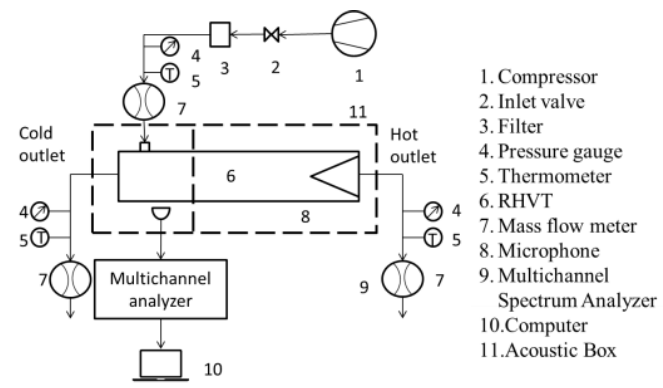

Figure 2. Schematic of the experimental setup

The air pressure is controlled by a valve placed before the inlet. The pressures were set at $10 \mathrm{psi}, 15 \mathrm{psi}, 20 \mathrm{psi}$ and 25 psi. This experiment was held in a thermodynamic laboratory at around $101 \mathrm{kPa}$ of atmospheric pressure.

The "anechoic" box is divided into two chambers to separate the hot tube and the cold tube. This is to ensure that only the sound produced on each side is recorded.

Fig. 3 shows the swirl generator used in this experiment. Four orifice diameters were applied: $2 \mathrm{~mm}, 3$ $\mathrm{mm}, 4 \mathrm{~mm}, 5 \mathrm{~mm}$ and $6 \mathrm{~mm}$.

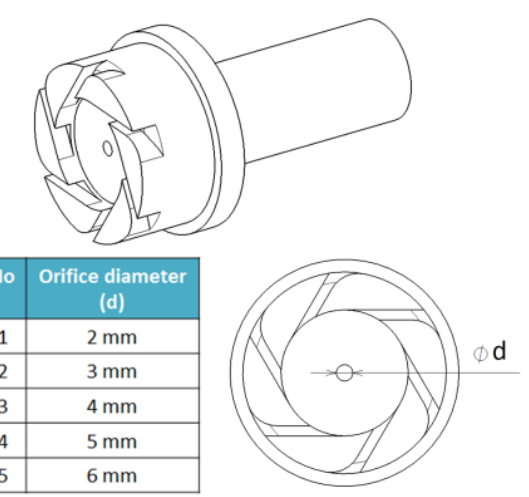

Figure 3. Drawing of the swirl generator

$\Delta T_{c}$ is the temperature difference between the air at the inlet $\left(T_{i}\right)$ and the cold air exiting from the cold tube $\left(T_{c}\right)$ (Eq. 1). This difference of temperatures represents the cooling capability of the device.

$$
\Delta T_{c}=T_{i}-T_{c}
$$

Mass fraction is the ratio between the outlet mass flow rate $\left(\dot{m}_{c}\right)$ and the inlet mass flow rate $\left(\dot{m}_{i}\right)$ (Eq. 2$)$. It represents the portion of air flowing through the cold outlet.

$$
\mu_{c}=\frac{\dot{m}_{c}}{\dot{m}_{i}}
$$

Isentropic efficiency $\left(\eta_{i s e n}\right)$ is calculated using the principle of adiabatic expansion of an ideal gas (Eq. 3), where $\gamma$ is the specific heat ratio (for air $\gamma=1.4), P_{a}$ and $P_{i}$ represent the atmospheric pressure and the inlet pressure of the air respectively.

$$
\eta_{\text {isen }}=\frac{T_{i}-T_{c}}{T_{i}\left(1-\left(\frac{P_{a}}{P_{i}}\right)^{\frac{\gamma-1}{\gamma}}\right)}
$$

The recorded sounds were processed using FastFourier Transform (FFT) to obtain the frequency spectrum of the signals. Main frequencies are then extracted and replaced by bars representing the frequency location and magnitude.

\section{Result And Discussion}

\subsection{Thermofluid analysis}

Fig. 4 shows the effect of orifice diameter to the isentropic efficiency RHVT. The isentropic efficiency gradually increases from orifice diameter of $2 \mathrm{~mm}$ to $4 \mathrm{~mm}$ then it decreases at higher diameters. Among five orifice diameters used, the diameter of $4 \mathrm{~cm}$ produces maximum efficiency (around 16\% - 18\%) for all four pressures applied at the inlet. The highest isentropic efficiency achieved is $17.88 \%$ for orifice diameter $4 \mathrm{~mm}$ at $15 \mathrm{psi}$ of inlet pressure. It is also observed that at $2 \mathrm{~mm}$ orifice diameter, the value of efficiency is almost the same for all inlet pressures.

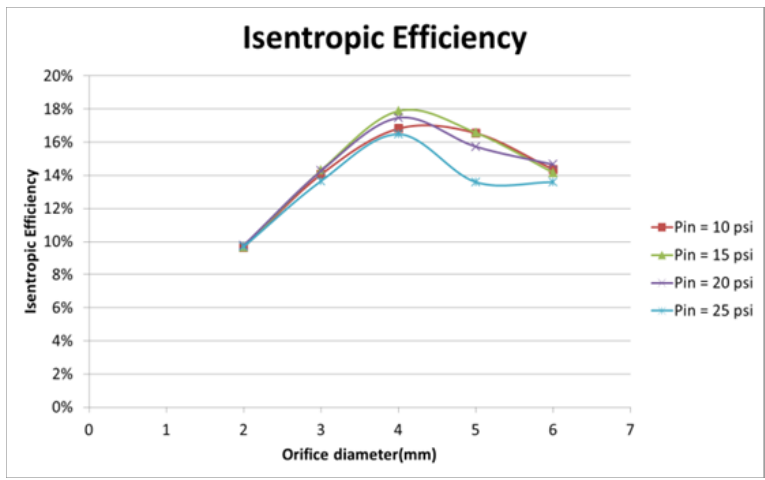

Figure 4. Isentropic efficiency vs orifice diameter.

Fig. 5 shows the variation of difference of temperature between the inlet and the cold outlet when the orifice diameter is varied. Four gage pressures were applied at 
the inlet. All curves give almost the same trend, it climbs from orifice diameter $2 \mathrm{~mm}$ to $4 \mathrm{~mm}$ then it descends at bigger diameters. Higher inlet pressure gives higher temperature difference for all diameters. The maximum temperature difference is obtained when the orifice diameter equals $4 \mathrm{~mm}\left(12^{\circ} \mathrm{C}\right.$ at $25 \mathrm{psi}$ inlet pressure $)$.

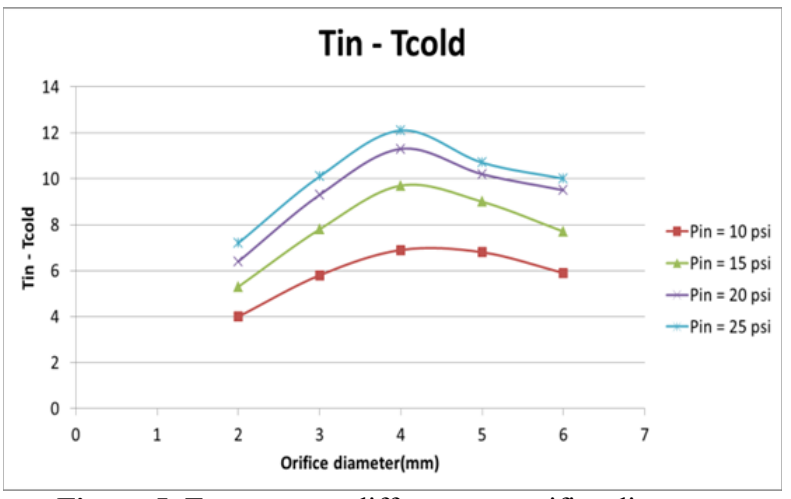

Figure 5. Temperature difference vs orifice diameter.

Fig. 6 represents the variation of cold mass fraction when the inlet pressure is varied at different orifice diameter. The results show that the cold mass fraction is almost constant at each orifice diameter. The mass fraction is higher when the orifice diameter is bigger, as it allows larger amount of air passing through it. The average cold mass fractions produced are $0.28,0.43,0.53$, 0.58 and 0.61 at orifice diameters of $2 \mathrm{~mm}, 3 \mathrm{~mm}, 4 \mathrm{~mm}$, $5 \mathrm{~mm}$ and $6 \mathrm{~mm}$ respectively.

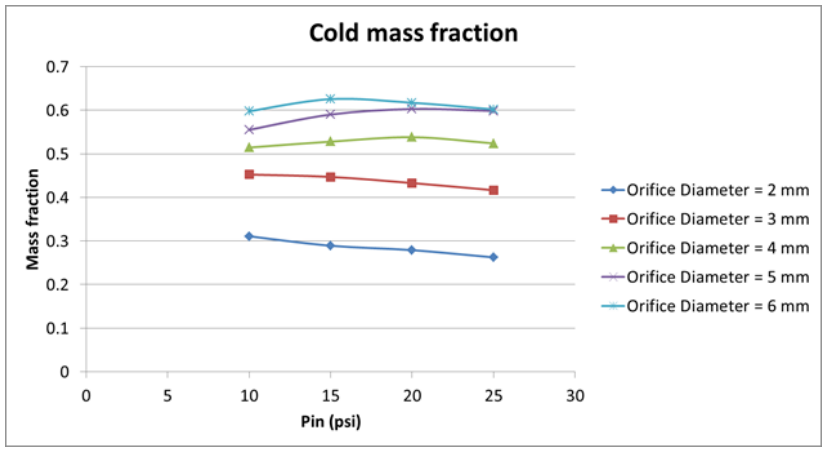

Figure 6. Cold mass fraction vs inlet pressure.

\subsection{Acoustic analysis}

Fig. 7 represents the sound signals produced at the cold tube. The sound is recorded for $80 \mathrm{~ms}$ using $2 \mathrm{~mm}$ orifice diameter ( $1^{\text {st }}$ column), $3 \mathrm{~mm}$ diameter ( $2^{\text {nd }}$ column), $4 \mathrm{~mm}$ diameter $\left(3^{\text {rd }}\right.$ column $)$ and $5 \mathrm{~mm}$ diameter $\left(4^{\text {th }}\right.$ column) at inlet pressures of $10 \mathrm{psi}\left(1^{\text {st }}\right.$ row $), 15 \mathrm{psi}\left(2^{\text {nd }}\right.$ row $), 20$ psi ( $3^{\text {rd }}$ row) and 25 psi ( $4^{\text {th }}$ row $)$. The signal produced by orifice diameter $6 \mathrm{~mm}$ is not included in this acoustic analysis as the frequency produced is very weak.

The signal produced at the cold tube mostly contains two categories of frequencies, i.e. low and high frequencies. The low frequency is produced by the surrounding noise, such as from the air-conditioning vent (around $1200 \mathrm{~Hz}$ ), compressor (around $2500 \mathrm{~Hz}$ ), etc. The use of "anechoic" box eliminates the noise coming from the air-conditioning system. However, as the compressor is always connected to the device through the inlet pipe, the noise coming from the compressor (when it is running) is more difficult to eliminate. During experiments, the compressor ran intermittently. The presence of the compressor noise can be noticed in figure 7 (c), (h), (j), (k), (l), (m), (n) and (o).

The magnitude of the Fourier Transform of the signals in Fig. 7 is presented in Fig. 8. It is obtained using FFT algorithm to show the frequency spectrum of the signals. The unit of the vertical axis is Pa representing the sound pressure. From the FFT results, some peaks present at different locations representing significant frequencies produced by the device captured at the cold tube for different orifice diameters. It is observed that when the inlet pressure increases (from top to bottom) the magnitude of the peaks increases. The position (the frequency) of the peaks does not seem to displace significantly when the same orifice diameter is used. For orifice diameter equals $2 \mathrm{~mm}$ one significant frequency appears at around $5920 \mathrm{~Hz}$ which is produced by the orifice. For larger diameters, more peaks appear. The highest magnitude still comes from the orifice and the others come from the right side of the orifice (the swirl generator and the hot tube) which are captured by the microphone located at the cold tube. This is confirmed by the acoustic analysis of the hot tube [7] which captured the same frequencies but with higher magnitudes (see Fig. 9). Except the frequency of the sound created by the orifice, other frequencies show higher in magnitude at the hot tube side. This indicates these sounds are produced inside the hot tube or closer to the hot tube. Returning back to Fig. 8, it is observed that for orifice diameter equals $3 \mathrm{~mm}, 4 \mathrm{~mm}$ and $5 \mathrm{~mm}$, the frequencies produced by the orifice are around $3630 \mathrm{~Hz}, 3300 \mathrm{~Hz}$ and $3130 \mathrm{~Hz}$ respectively, which are decreasing following the increase of the orifice diameter.

If the peaks that are present in the FFT results (Fig. 8) are replaced by bars with the same order of magnitude (after removing the low frequencies coming from the surrounding noise), the frequency representation becomes the one presented in Figure 10. This made the analysis clearer. Each acoustic signal at different inlet pressure with different orifice diameter is now represented by a set of bars located at specific frequencies carrying different magnitude. The term "signature" can be used for each representation. These cold tube signatures represent the thermofluid performance shown in Figures 4, 5 and 6 from the cold tube point of view. For example, the signature from $2 \mathrm{~mm}$ orifice diameter containing frequencies around $5920 \mathrm{~Hz}$ and $19600 \mathrm{~Hz}$ with magnitudes of $0.0036 \mathrm{~Pa}$ and $0.0009 \mathrm{~Pa}$ respectively (Fig. 10 (i)) represents the isentropic efficiency of $10 \%$ (see Fig. 4), temperature difference of $6.4^{\circ} \mathrm{C}$ (see Fig. 5) and a cold mass fraction of 0.279 (see Fig. 6). Another example, the signature of $4 \mathrm{~mm}$ orifice diameter at $20 \mathrm{psi}$ containing frequencies around $3312 \mathrm{~Hz}, 7750 \mathrm{~Hz}, 16362$ $\mathrm{Hz}, 18512 \mathrm{~Hz}$ and $19950 \mathrm{~Hz}$ with magnitudes of 0.00163 $\mathrm{Pa}, 0.00067 \mathrm{~Pa}, 0.00087 \mathrm{~Pa}, 0.00083 \mathrm{~Pa}$ and $0.00088 \mathrm{~Pa}$ (Figure $10(\mathrm{k})$ ) represents the isentropic efficiency of $17.47 \%$, temperature difference of $11.3^{\circ} \mathrm{C}$ and a cold mass fraction of 0.538 . For other signatures, the thermofluid performance can be consulted directly in 
Figure 10. These cold tube signatures complement the hot

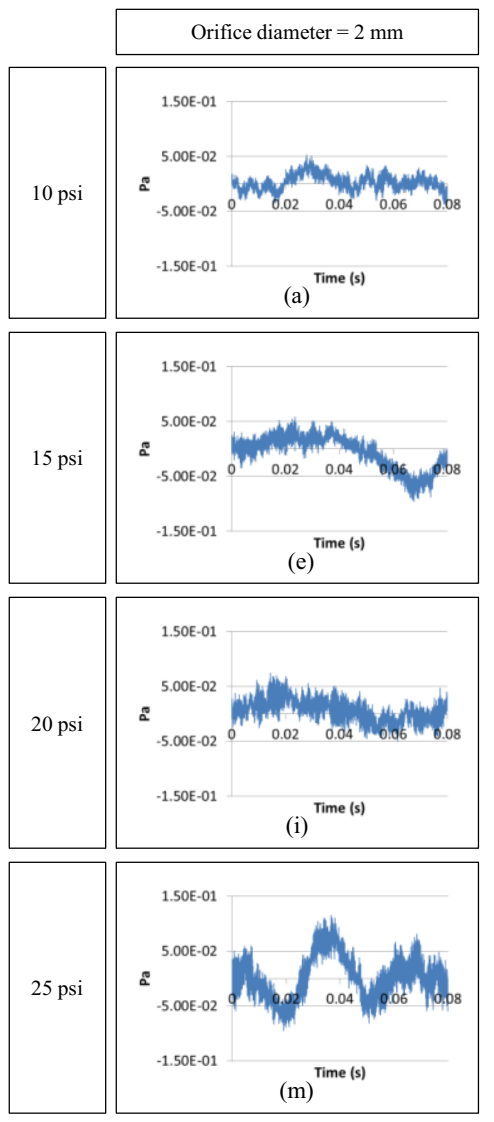

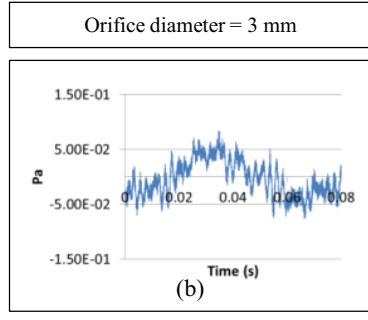
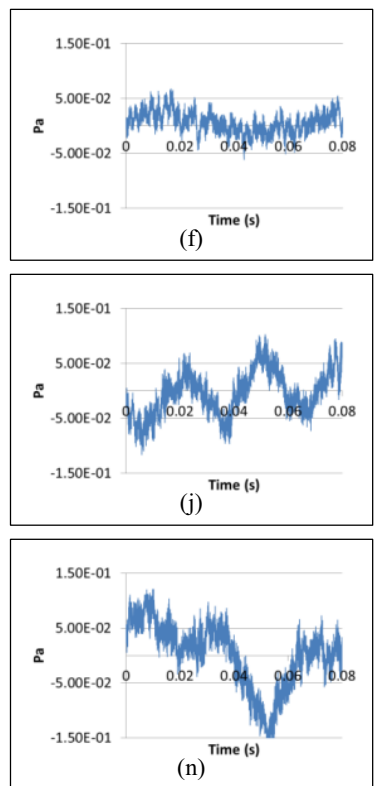

tube signatures from the reference [7].

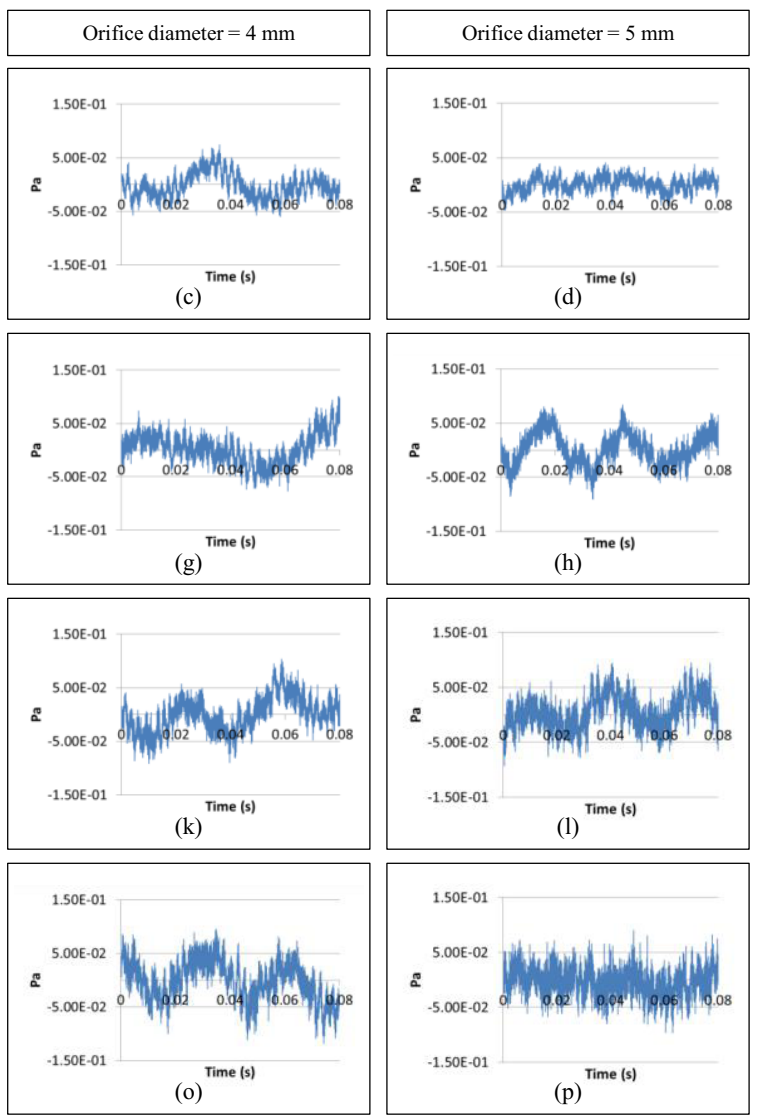

Figure 7. Signal produce by the RHVT with different orifice diameters at different inlet pressures.
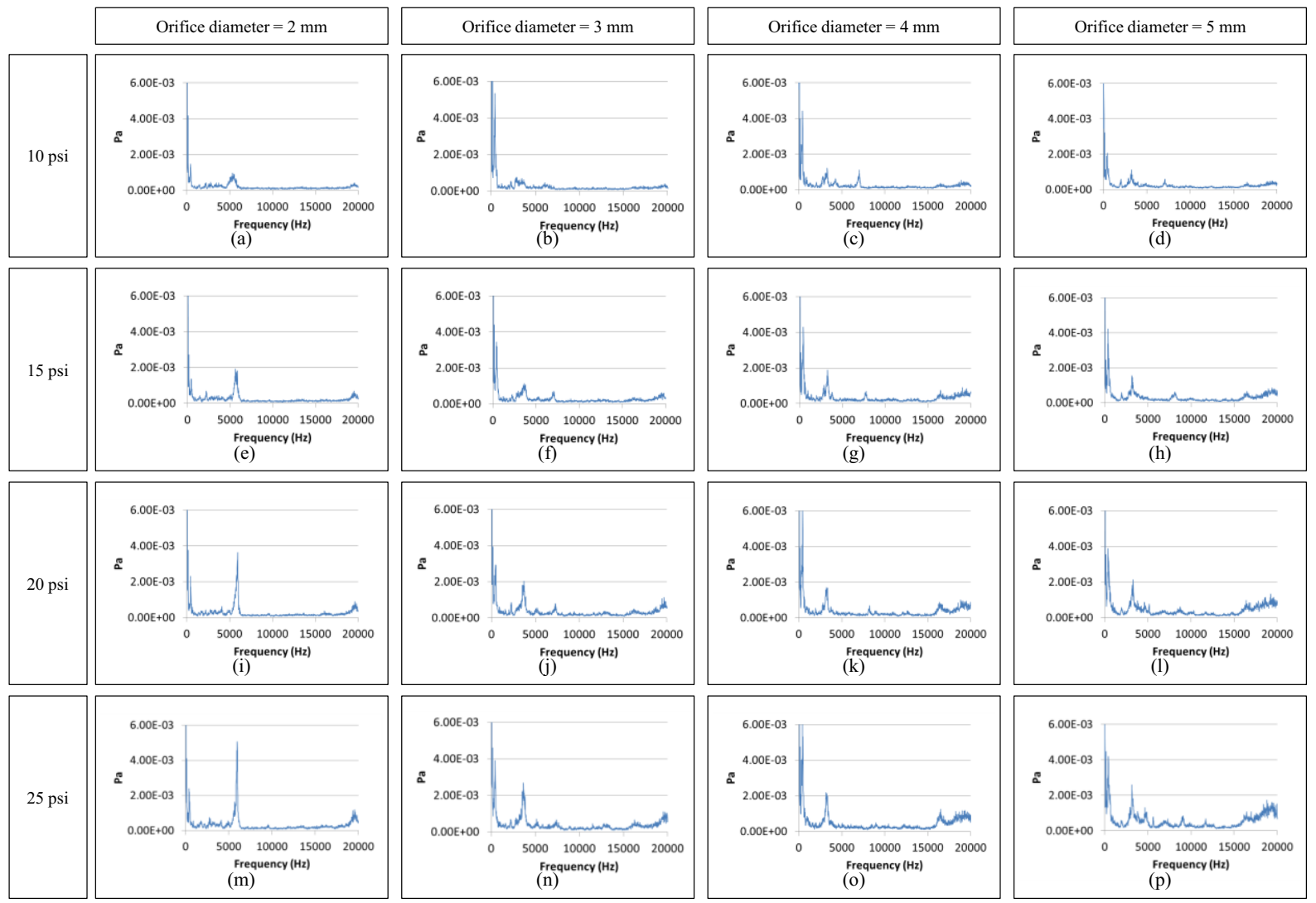

Figure 8. Magnitude of FFT of the signals in Figure 7. 


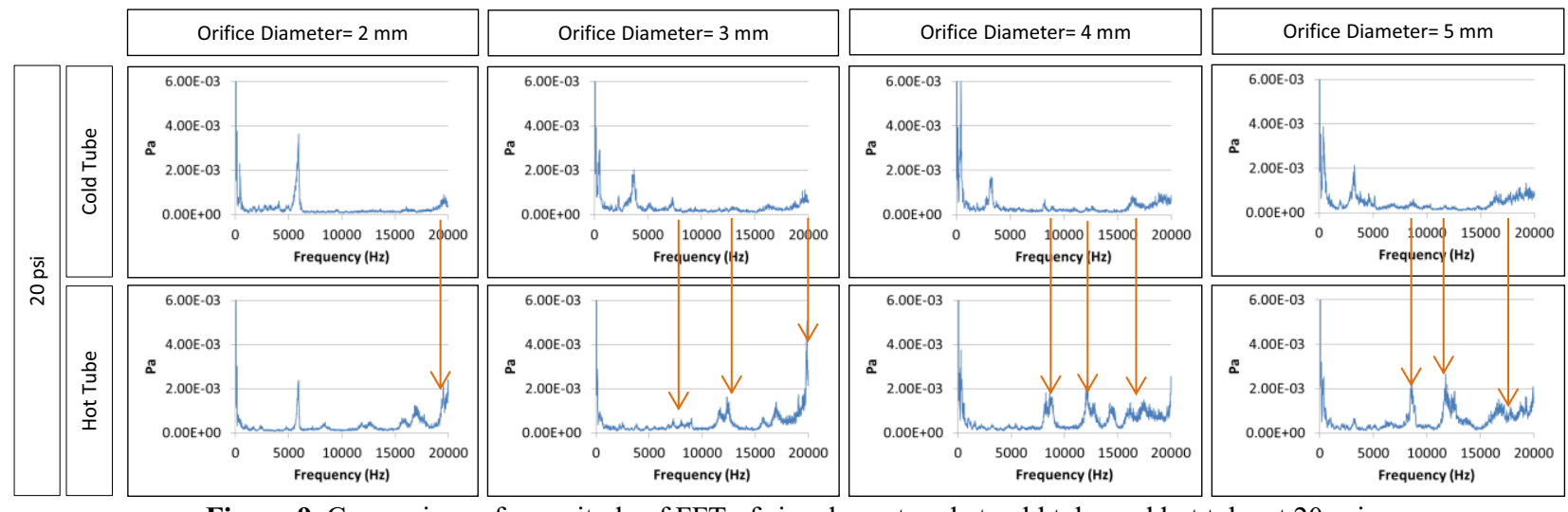

Figure 9. Comparison of magnitude of FFT of signals captured at cold tube and hot tube at 20 psi.
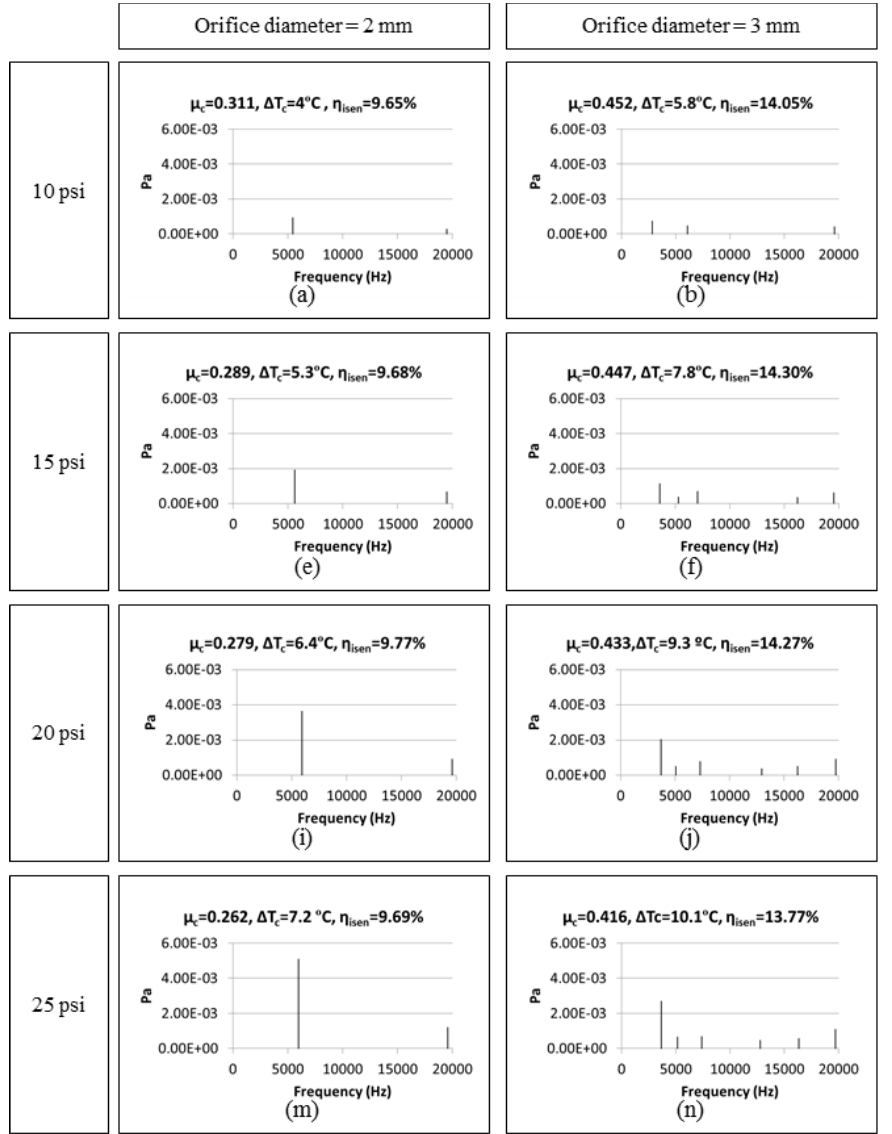
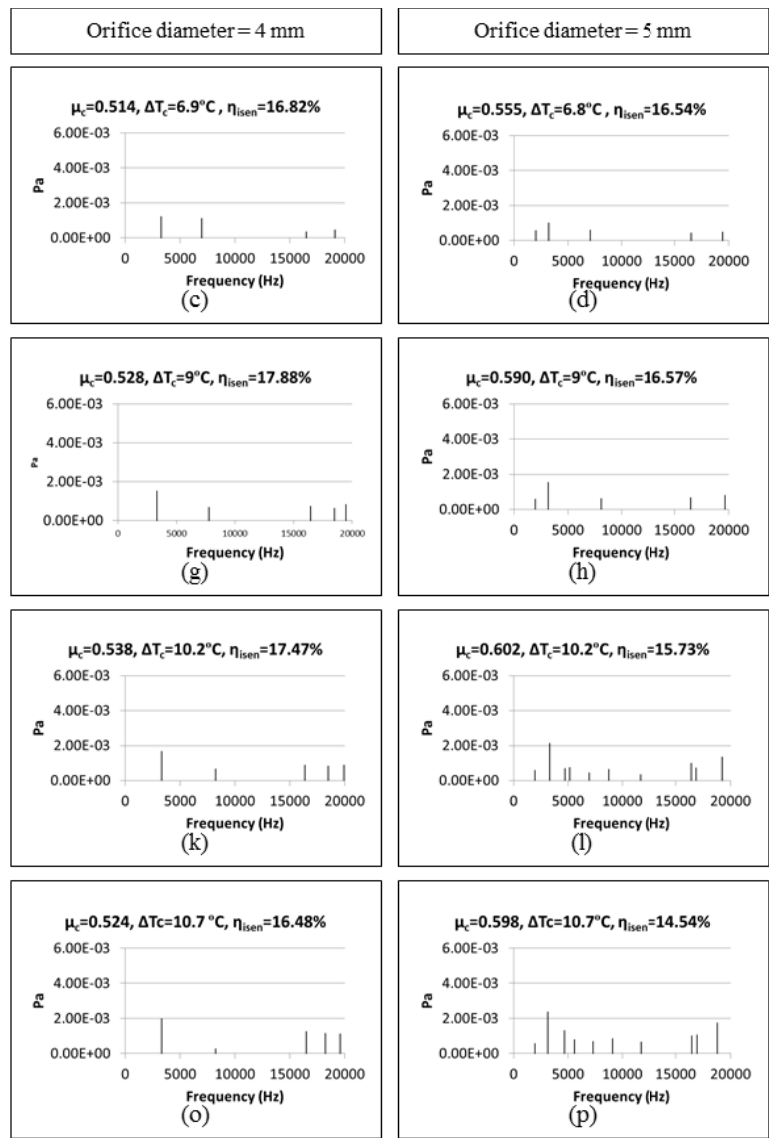

Figure 10. Main frequencies extracted from signals in Fig. 8.

\section{Conclusions}

In this study, the effect of orifice diameter on the thermofluid performance of the RHVT and the acoustic signal produced on the cold side of the tube is investigated. The investigation has been conducted experimentally and simple signal processing using FFT has been applied. It is observed that different orifice diameter produces different set of acoustic frequencies. This set of frequencies forms a signature of the signal from the cold tube point of view that can be associated to the thermofluid performance of the device. These cold tube signatures complement the hot tube signatures, so that the performance of the RHVT can be represented by the acoustic signatures (both cold and hot tubes) produced by the device.

\section{Acknowledgements}

Authors would like to thank the Malaysian Ministry of Higher Education (MOHE), the Research Management Centre (RMC) and the Faculty of Mechanical Engineering, Universiti Teknologi MARA (UiTM) for providing fund and support for this research under Fundamental Research Grant Scheme (FRGS), File No.: 600-RMI/FRGS 5/3 (74/2014). 


\section{References}

1. N. Ismail, W. Wisnoe, M. F. Remeli, Experimental Investigation of Orifice Diameter, Swirl Generator and Conical Valve Shape to the Cooling Performance of Ranque-Hilsch Vortex Tube, Applied Mechanics and Materials 510, 174-178 (2014).

2. H. R. Thakare, A. Monde, A. D. Parekh, Experimental, computational and optimization studies of temperature separation and flow physics of vortex tube: A review, Renewable and Sustainable Energy Reviews 52,1043-1071 (2015).

3. S. U. Nimbalkar, M. R. Muller, An experimental investigation of the optimum geometry for the cold end orifice of a vortex tube, Applied Thermal Engineering, 29(2-3), 509-514, (2009).

4. Y. Istihat, M. H. Sabri, W. Wisnoe K. M. Abd Rahman, Flow Visualization in a Ranque-Hilsch Vortex Tube, (submitted to Journal of Mechanical Engineering).

5. W. Wisnoe, K.M. Abd Rahman, Y. Istihat, D. V. Natarajan, Thermofluid-Acoustic Analysis of a Ranque-Hilsch Vortex Tube, 3rd International
Conference on System-integrated Intelligence: New Challenges for Product and Production Engineering, SysInt, Procedia Technology, (2016)

6. M. Kurosaka, Acoustic streaming in a swirling flow and the Ranque-Hilsch (vortex tube) effect, Journal of Fluid Mechanics, 124, 139-172, (1982).

7. K. M. Abd Rahman, W. Wisnoe, Y. Istihat, D. V. Natarajan, Acoustic analysis on different orifice diameter of a Ranque-Hilsch Vortex Tube (hot side), Mechanical Engineering \& Science Postgraduate International Conference, (2016).

8. Y. Istihat, W. Wisnoe, "Wavelet Transform of Acoustic Signal From A Ranque-Hilsch Vortex Tube, IOP Conference Series: Materials Science and Engineering, 88, (2015).

9. N. Ismail, W. Wisnoe, M. F. Remeli, Experimental Investigation on the Effect of Orifice Diameter and Inlet Pressure to the Ranque-Hilsch Vortex Tube Performance. Applied Mechanics and Materials, 465-466, 515-519 (2014) 